\title{
Efficacy of first-line systemic treatment in correlation with BRAF V600E and different KRAS mutations in metastatic colorectal cancer - a single institution retrospective analysis
}

\author{
Martina Rebersek ${ }^{1}$, Marko Boc ${ }^{1}$, Petra Cerkovnik², Jernej Benedik ${ }^{1}$, Zvezdana Hlebanja ${ }^{1}$, \\ Neva Volk ${ }^{1}$, Srdjan Novakovic ${ }^{2}$, Janja Ocvirk ${ }^{1}$ \\ ${ }^{1}$ Department of Medical Oncology, ${ }^{2}$ Department of Molecular Diagnostics, Institute of Oncology Ljubljana, Ljubljana, Slovenia
}

Received 4 August 2011

Accepted 20 September 2011

Correspondence to: Martina Reberšek, Department of Medical Oncology, Institute of Oncology Ljubljana, Zaloška 2, 1000 Ljubljana Slovenia. Phone: +386 15879 220; Fax: +3861 5879 400; E-mail: mrebersek@onko-i.si

Disclosure: No potential conflicts of interest were disclosed.

Background. KRAS mutation status in codons 12 and 13 is recognized as a predictive factor for resistance to antiEGFR monoclonal antibodies. Despite having a wild type KRAS (wt-KRAS), not all patients with wt-KRAS respond to anti-EGFR antibody treatment. Additional mechanisms of resistance may activate mutations of the other main EGFR effectors pathway. Consequently, other molecular markers in colorectal cancer are needed to be evaluated to predict the response to therapy.

Patients and methods. In this retrospective study, objective responses (OR), time to progression (TTP), overall survival (OS) were analyzed in 176 metastatic colorectal cancer (MCRC) patients treated with first-line chemotherapy in combination with monoclonal antibodies in respect of KRAS status in codons 12 and 13 and BRAF mutational status. Results. The KRAS mutations were found in 63 patients (35.8\%), the KRAS mutation in codon 12 in 53 patients (30.1\%) and the KRAS mutation in codon 13 in 10 patients (5.7\%). The BRAF V600E mutation was detected in 13 of 176 patients (7.4\%). In the subgroup of mCRC patients having wt-KRAS and wild type BRAF (wt-BRAF), the objective response rates were higher (OR 54.0\%,CR 14.7\%, PR 39.3\%) than in the patients with wt-KRAS and mt-BRAF (OR 38.5\%,CR 15.4\%, PR $23.1 \%)$, the difference was not statistically significant $(p=0.378)$. Median OS in patients with wt-KRAS wt-BRAF, and in patients with wt-KRAS mt-BRAF, was 107.4 months and 45 months, respectively. The difference was statistically significant $(p=0.042)$. TTP in patients with wt-KRAS wt-BRAF, and in patients with wt-KRAS mt-BRAF, was 16 months and 12 months, respectively. The difference was not statistically significant $(p=0.558)$.

Conclusions. Patients with BRAF V600E mutation have statistically significantly worse prognosis than the patients with Wt-BRAF and progress earlier during treatment. The definitive role of the BRAF V600E mutation as a prognostic and predictive factor for the response to anti-EGFR monoclonal antibodies needs to be analyzed in large prospective clinical studies.

Key words: metastatic colorectal cancer; KRAS; BRAF; prognostic factors

\section{Introduction}

Colorectal cancer (CRC) is the fourth most common cancer and one of the leading causes of cancer death in the world. It is the most common cancer in Slovenia and, according to the Cancer Registry of Slovenia, 1279 new patients were diagnosed with CRC in $2007 .{ }^{1}$ The majority of patients need combined modality treatment and carful posttreatment surveillance is necessary to offer patient an optimal treatment approach.,.$^{2,3}$ Metastatic disease is still incurable, with $5 \%$ five-year survival without treatment. With the introduction of new chemotherapy, using oxaliplatin and irinotecan in the current management of metastatic disease, in combination with biologicals, targeting epidermal 
growth factor- mediated growth regulatory pathway and the vascular endothelial growth factormediated angiogenesis pathway, we can prolong the progression-free survival (PFS) and overall survival (OS) of these patients. ${ }^{4-8}$ In selected patients with appropriate combination of therapy and surgery we can achieve approximately a 50\% five-year survival.

The development of CRC is a multistep process which accumulates different gene mutations, chromosomal abnormalities and epigenetic changes. ${ }^{9,10}$ The mutations within KRAS proto-oncogen, predominately within codons 12 and 13, activate RAS/RAF signalling and are thought to occur early in carcinogenesis of CRC. The KRAS status is the first molecular marker to predict the response to anti-EGFR monoclonal antibodies cetuximab and panitumumab in metastatic CRC (mCRC) patients, and it needs to be determined before deciding in favor of treatment with anti-EGFR antibodies. As the KRAS mutations occur early in CRC formation, there is a high concordance between the KRAS mutations of primary tumour and metastases, which was confirmed in previous studies. ${ }^{11-13}$ In a recent retrospective study, de Roock with his colleagues raised the possibility that the patients with the KRAS mutation in codon 13 might have benefited from anti- EGFR antibodies treatment. ${ }^{14}$ The mutations in KRAS gene are found in approximately 30 to $40 \%$ of mCRC patients, reported in previous literature, but only 40 to $60 \%$ of these patients with wt-KRAS will respond to anti-EGFR antibodies treatment. ${ }^{15,16}$ Therefore, other molecular markers downstream of EGFR in the RAS/RAF/MAPK pathway and other effector pathways are found to be involved to predict the response to specific systemic therapy.

The BRAF gene encodes a serine/threonine protein kinase of the RAS/RAF/MEK/ERK kinase pathway and it is also involved in CRC carcinogenesis. ${ }^{9,10}$ The most common mutation of the BRAF gene is $\mathrm{V} 600 \mathrm{E}$ which is found in approximately 5 to $9 \%$ of mCRC. ${ }^{17,18}$ The same was reported in our previous study carried on Slovenian patients with CRC where the BRAF V600E mutation was found in $5.1 \%$ of patients. ${ }^{19}$ Previous retrospective studies suggested that mt-BRAF was a marker of resistance to anti-EGFR therapy and that the patients with mt-BRAF had significantly shorter PFS and OS than the patients with wt-BRAF tumours. ${ }^{20}$ The mutations in the KRAS and BRAF genes have been reported to be mutually exclusive..$^{21,22}$ In the retrospective analysis by Fariña- Sarasqueta et al., it was also shown that the BRAF V600E mutation was an independent prognostic factor for the survival of patients with colon cancer in stages II and III, while the KRAS mutations did not have any effect on the overall survival of these patients. They concluded that the prognostic role of the KRAS mutations in an adjuvant setting has to be determined. ${ }^{23}$ In recent clinical studies, it was published that the BRAF V600E mutation in metastatic colorectal cancer is conferred to a poor prognosis regardless of treatment, but these patients may have some benefit from the treatment with cetuximab in combination with chemotherapy as the first-line therapy, but not when used in the patients in whom the disease has progressed after the first-line therapy. ${ }^{17}$

The aim of this retrospective study was to analyze objective responses, time to progression and overall survival of the patients with metastatic colorectal cancer treated with first-line systemic therapy in respect of KRAS and BRAF status.

\section{Patients and methods}

\section{Patients}

In the study, 176 patients with histologically confirmed metastatic colorectal cancer (mCRC), primarily metastatic or progressed during or after adjuvant therapy were retrospectively analyzed. They were treated according to the national and NCCN guidelines, including performance status of patients and comorbidity. They were treated with chemotherapy, including fluoropirimidins, capecitabine or 5-fluorouracil (5- FU), oxaliplatin or irinotecan in combination with biologicals, bevacizumab or cetuximab in respect of previously determined KRAS status. The treatment was continued according to the RECIST criteria, until the planned operation or until the progression of disease or toxicity occurred.

\section{Methods and assessment of response}

All relevant data from medical files were collected and entered into the data base. Baseline data was analyzed with regard to age, sex, primary site (colon and rectum), number and location of metastases. Efficacy was evaluated according to the Response Evaluation Criteria in Solid Tumours (RECIST, version 1.1) by using computed tomografy (CT) scans, magnetic resonance scans, abdominal ultrasound, chest X-ray, bone scans, clinical examination and laboratory tests. ${ }^{24}$ The study was conducted in the conformance with the principles of the Declaration of Helsinki. 


\section{Molecular analysis of KRAS and BRAF mutations}

DNA for molecular analysis was extracted from formalin-fixed, paraffin-embedded tumour tissue of primary tumours or metastases with at least $70 \%$ of tumour cells. TheraScreen KRAS Mutation $\mathrm{Kit}^{\circledR}$ (Roche Applied Science, Mannheim, D) was used to determine seven most common mutations in codons 12 and 13 of the KRAS gene. The V600E mutation in BRAF was detected by end-point genotyping using the TaqMan MGB probes (Applied Biosystems, Warrington, UK) as described previously. ${ }^{19}$ The mutation V600E in BRAF in positive tumour samples was confirmed by direct sequencing after amplification of the exon 15 of the BRAF gene. ${ }^{19}$

\section{Statistical analysis}

The primary end-points of the analysis were overall response rate (ORR), based on RECIST criteria, overall survival (OS) and time to progression (TTP) according to the KRAS and BRAF status.

The $\chi^{2}$-test was used to compare ORR, OS and TTP between groups, with $95 \%$ confidence intervals (CI) calculated for the medians. OS and TTP were estimated by using Kaplan-Meier Estimates and compared using the log-rank test. TTP was measured in all patients from the beginning of the first-line systemic chemotherapy to the first evidence of progression. The duration of survival was calculated from the beginning of systemic treatment until the date of death. $\mathrm{p}$ value $<0.05$ was considered statistically significant. Statistical data were obtained using the SPSS software package PASW statistics 18.0.

\section{Results}

\section{Patients' characteristics}

In total, 176 patients with $\mathrm{mCRC}$ who received first-line therapy between May 2005 and October 2010 were included in the retrospective analysis. The cut-off date for the present analysis was April 2011. All patients were treated at the Institute of Oncology Ljubljana, all were Caucasian. The median age was 62 years (range 27-86 years) and the majority of the patients were males (61.4\%). Most of the patients had metastatic colon cancer $(71.4 \%)$. One hundred and four patients had primary metastatic disease $(59.1 \%)$. The most common sites of metastases were liver and lung. The most com-
TABLE 1. Baseline and disease characteristic of patients

\begin{tabular}{|c|c|}
\hline Caracteristics & Patients, $n=176(\%)$ \\
\hline \multicolumn{2}{|l|}{ Gender } \\
\hline Male & $108(61.4)$ \\
\hline Female & $68(38.6)$ \\
\hline \multicolumn{2}{|l|}{ Age(years) } \\
\hline Median & 62 \\
\hline Range & $(27-86)$ \\
\hline \multicolumn{2}{|l|}{ WHO PS* } \\
\hline 0 & $126(71.6)$ \\
\hline 1 & $50(28.4)$ \\
\hline \multicolumn{2}{|c|}{ Primary tumour localization, } \\
\hline Colon & $125(71)$ \\
\hline $\begin{array}{l}\text { Rectum } \\
\text { Metastatic site }\end{array}$ & $51(28)$ \\
\hline Liver & $68(38.6)$ \\
\hline Lung & $11(6.3)$ \\
\hline Liver and lung & $12(6.8)$ \\
\hline Other & $85(49.3)$ \\
\hline \multicolumn{2}{|l|}{ KRAS status } \\
\hline KRASW & $113(64.2)$ \\
\hline KRASm 12 & $53(84.0)$ \\
\hline KRASm 13 & $10(16.0)$ \\
\hline \multicolumn{2}{|l|}{ BRAF status } \\
\hline BRAFW & $163(92.6)$ \\
\hline BRAFm & $13(7.4)$ \\
\hline
\end{tabular}

*WHO PS- World Health Organization performance status

mon therapies the patients received were irinotecan, capecitabine with bevacizumab $(29.5 \%)$ and oxaliplatin, capecitabine with cetuximab $(22.1 \%)$. Twenty-four patients $(13.6 \%)$ were treated only with chemotherapy, capecitabine in monotherapy, or with fluoropirimidines in combination with oxaliplatin or irinotecan. Patients' baseline and disease characteristics are shown in Table 1.

KRAS mutations were found in 63 patients $(35.8 \%)$, to be more precise, the KRAS mutation in codon 12 in 53 patients $(84.0 \%$ ) and the KRAS mutation in codon 13 in 10 patients (16.0\%). The BRAF V600E mutation was detected in 13 of 176 patients $(7.4 \%)$.

The mutations of the KRAS or BRAF gene were detected in total in 76 patients (43.4\%) (Table1).

\section{Efficacy}

The response rates according to RECIST criteria with regard to the KRAS and BRAF status are shown in Table 2. The overall response rates in pa- 


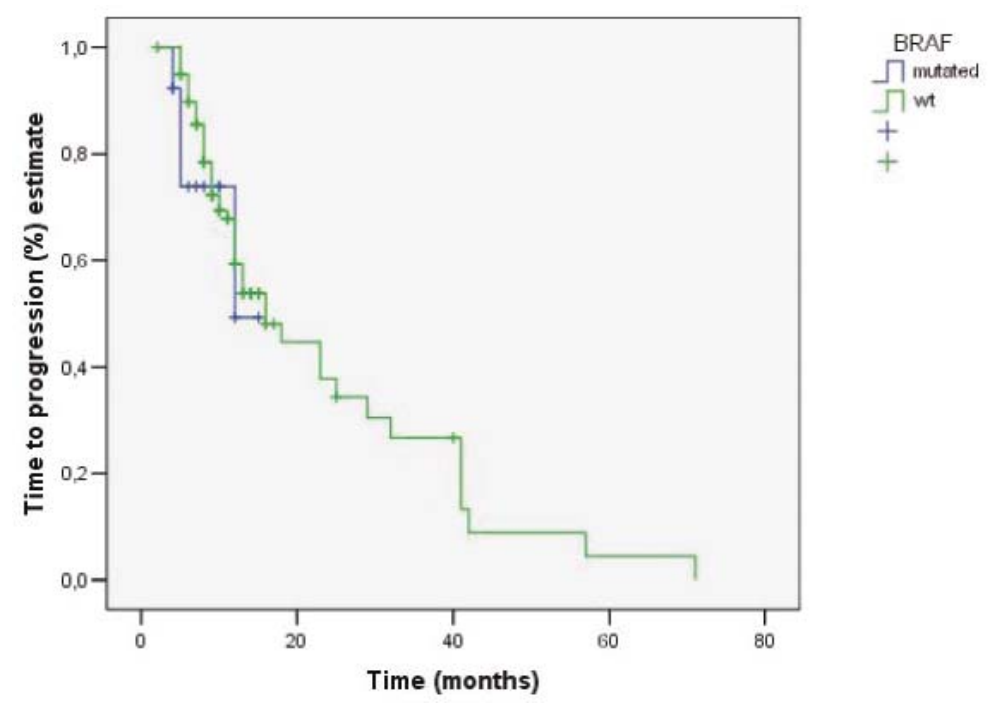

FIGURE 1. Time to progression in patients with wt-KRAS/wt-BRAF and wt-KRAS/mt-BRAF.

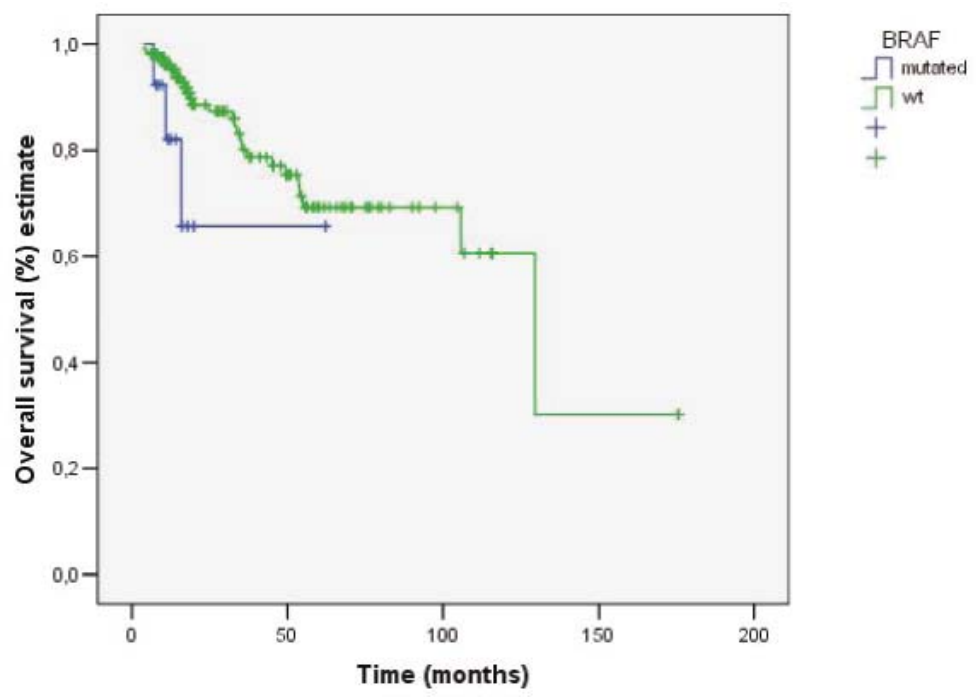

FIGURE 2. Overall survival in patients wt-KRAS/wt-BRAF and wt-KRAS/mt-BRAF.

tients with wt-KRAS and wt-BRAF and in patients with wt- KRAS and mt- BRAF were CR $14.7 \%+$ PR $39.3 \%+$ SD $35.5 \%$ and CR $15.4 \%+$ PR $23.1 \%+$ SD $46.1 \%$ respectively. The objective response rates in the group of patients with wt-KRAS and wt-BRAF tumours were $54.0 \%$ (CR $14.7 \%$, PR 39.3\%), while in the group of patients with wt-KRAS and mtBRAF were $38.5 \%$ (CR $15.4 \%$, PR $23.1 \%$ ). The difference was not statistically significant $(p=0.378)$. The median OS in the group of patients with wtKRAS and wt-BRAF tumours was 107.4 months (95\% CI: 82- 132.9 months) and in the group of patients with wt-KRAS and mt-BRAF tumours 44.9 months (95\% CI: 28.4- 61.5 months) (Figure 1). The difference in median OS between those two groups was statistically significant $(p=0.042)$. TTP in the group of patients with wt-KRAS and wt-BRAF tumours and in the group of patients with wt-KRAS and mt-BRAF tumours was 16 months (95\% CI: 10.7- 21.2 months) and 12 months (95\% CI: 4.0- 15.0 months), respectively (Figure 2). It was not statistically significant $(\mathrm{p}=0.558)$.

In the KRAS mutation subgroups, the objective response rate of 53 patients with the mutation in codon 12 was $47 \%$ (CR 20.7\%, PR 26.4\%) and, in 10 patients with the mutation in codon 13 , the objective response was 33\% (CR 11.1\%, PR 22.2\%). The difference was not statistically significant $(\mathrm{p}=0.08)$. TTP in the patients with the mutation in codon 12 and the patients with the mutation in codon 13 was 13.5 months (95\% CI: 9- 18 months) and 9.3 months (95\% CI: 5.1- 13.5 months), respectively. The difference was not statistically significant $(p=0.106)$.

Surgical resection of liver metastases was performed in 47/176 patients (26.7\%); more specifically, in 31 patients with wt-KRAS tumours and in 16 patients with mt-KRAS tumours. R0 resection was achieved in 38/176 patients (21.6\%), of whom 37 patients had wt-BRAF and only one had mt-BRAF tumour.

\section{Discussion}

In our study population, the KRAS mutations in codons 12 and 13 were found in $35.8 \%$ of patients, in most of them in codon 12; while the mutation $\mathrm{V} 600 \mathrm{E}$ in BRAF gene was detected in 13 patients $(7.4 \%)$. The results of testing are comparable with those previously reported, where the KRAS mutations were found in 30 to $40 \%$ and the BRAF V600E mutation in 5 to $9 \%$ of the patients. ${ }^{11-13,17-19}$

The presented data demonstrate that the patients with the BRAF V600E mutation have worse prognosis than the patients with wt-BRAF tumour and progress early during treatment. The patients with wt-BRAF tumours have higher response rates than the patients harbouring the BRAF V600E mutation, but the difference was not statistically significant. One third of the patients with wt-KRAS or mt-BRAF tumour still respond to the treatment, alluding that the BRAF status is not predictive for the response to anti-EGFR antibody therapy. This was also reported in previously published analyses and, in recently published retrospective metaanalysis of the CRYSTAL and OPUS studies, it was also concluded, that the patients with BRAF muta- 
TABLE 2. Response rates in KRAS wild type patients according to BRAF status in first-line therapy

\begin{tabular}{|c|c|c|c|}
\hline & WKRAS & WKRAS/WBRAF & WKRAS/mBRAF \\
\hline $\begin{array}{l}\text { Overall response rate } \\
\text { (CR+ PR), } n(\%)\end{array}$ & $93(52.8)$ & $88(54.0)$ & $5(38.5)$ \\
\hline $\begin{array}{l}\text { Disease control rate } \\
(C R+P R+S D), n(\%)\end{array}$ & $157(89.2)$ & $146(89.5)$ & $11(84.6)$ \\
\hline$C R$ & $26(14.8)$ & $24(14.7)$ & $2(15.4)$ \\
\hline PR & $67(38.0)$ & $64(39.3)$ & $3(23.1)$ \\
\hline SD & $64(36.4)$ & $58(35.5)$ & $6(46.1)$ \\
\hline PD & $19(10.8)$ & $17(10.5)$ & $2(15.4)$ \\
\hline $\begin{array}{l}\text { Median OS, months } \\
\text { estimate }\end{array}$ & 129.4 (95\% Cl: 52.4- 206.4) & 107.4 (95\% Cl: 82- 132.9)* & 44.9 (95\% Cl: 28.4- 61.5) * \\
\hline $\begin{array}{l}\text { Median TTP, months } \\
\text { estimate }\end{array}$ & 15.9 (95\% Cl: 10.8- 21.0) & 16.0 (95\% Cl: 10.7-21.2)** & 12.0 (95\% Cl: 4.0-15.0) ** \\
\hline
\end{tabular}

$* p=0.042 \quad * * p=0.558$

tion might have also benefited from the treatment with anti-EGFR antibodies. ${ }^{17,18,21-23,25}$ At this point it should be highlighted that not all patients in our retrospective analysis with wt-KRAS received cetuximab-based first-line systemic therapy; the therapy was selected in accordance to the patients' baseline characteristics, the purpose of treatment or planned operation for metastases.

The difference in TTP between the patients with wt-KRAS and wt-BRAF tumours and the patients with wt-KRAS and mt-BRAF tumours was 4 months. The difference was not statistically significant, probably due to our small group of patients and, consequently, small proportion of the patients with BRAF mutation. The comparison of median OS of those two groups showed a statistically significant difference which was also accompanied with a better prognosis of patients with wt-KRAS and wt-BRAF tumour. These results are comparable with those reported earlier. ${ }^{17,20,21}$ The results of retrospective pooled analysis from randomized CRYSTAL and OPUS trials showed that cetuximab as the first-line chemotherapy based on irinotecan or oxaliplatin significantly improved OS, ORR and PFS the in patients with wt-KRAS tumours. According to the results of the same meta-analysis, the patients with BRAF mutations also appeared to have benefited from cetuximab as the first-line systemic treatment. ${ }^{25,26}$

In our retrospective study, the KRAS mutations were most frequently detected in codon 12. This is in accordance with the results of our previous study. ${ }^{19}$ Comparing the patients having KRAS mutations in codon 12 with the ones having the muta- tion in codon 13 after the treatment with chemotherapy and bevacizumab, the response rates were higher in the patients with the mutations in codon 12. Nevertheless, the differences in response rates, OS and TTP between these two groups were not statistically significant; we assume that the groups of patients were too small. In the contrast, in their retrospective study, De Roock et al. showed that the patients with the mutation in codon 13 KRAS who were treated with cetuximab had better overall and progression-free survival than the patients with other KRAS mutations and might have benefited from the treatment with cetuximab. ${ }^{14}$ In an abstract recently published in the 2011 ASCO Annual Meeting Proceedings, Tejpar et al. retrospectively analyzed the influence of KRAS G13D mutations on the efficacy of treatment with cetuximab as the first-line systemic therapy and compared it with the pooled results of randomized studies CRYSTAL and OPUS. The patients with the KRAS mutation in codon 13 had a much lower treatment effect compared to the patients with wt-KRAS tumours and might have nevertheless benefited from treatment with cetuximab. ${ }^{27}$

Although not studied in our retrospective analysis, other KRAS mutations were also reported to predict the response to anti- EGFR monoclonal antibodies. The results of a small study of 74 patients, conducted by Loupakis with his colleagues, suggested that rare KRAS mutations in codon 61 and in codon 146 might also be responsible for in the treatment resistance to anti-EGFR monoclonal antibodies. ${ }^{28,29}$ In contrast, in their large retrospective analysis, De Roock et al. concluded that the codon 
146 mutations did not affect the response to cetuximab and that the patients with codon 61 mutant tumours had lower response rate. ${ }^{20}$ According to the analysis of other mutations, they proposed testing of KRAS status, if not mutated, then of BRAF and NRAS status, and PIK3CA exon 20 mutation in order to improve the objective response up to $40 \%$ in selected patients.

In our retrospective study, $26.7 \%$ of patients, all with KRAS wild-type tumours, who had previously unresectable liver-only metastases, underwent surgical resection after systemic therapy, with R0 resection achieved in 38 patients $(21.6 \%)$; one of those was patient with the BRAF V600E mutation. Although it is difficult to make any comparison, because our patients were not selected according to specific systemic therapy, these results are comparable with those reported in previous studies claiming that 19 to $23 \%$ patients treated with bevacizumab- and irinotecan-based chemotherapy and with previously unresectable liver-only metastases underwent resection..$^{30-32}$ In a recently published clinical study BOXER, where the patients with unresectable liver-only metastases were treated with oxaliplatin, capecitabine and bevacizumab, $\mathrm{R} 0$ resection was achieved in $40 \%$ of patients. ${ }^{33}$ The proportion of patients with resected liver metastases in our retrospective study was higher than that reported in earlier studies including the patients with previously unresectable liver-only metastases and treated with cetuximab in combination with irinotecan- or oxaliplatin-based chemotherapy; resection was achieved in 4 to $10 \% .{ }^{34,35}$ In the randomized phase II CELIM study, in which the patients with liver-only metastases were treated with irinotecan- or oxaliplatin-based chemotherapy with cetuximab as the first-line systemic therapy, the proportion of R0 resection was higher; it was achieved in $34 \%$ of patients. ${ }^{36}$ In another phase II POCHER trial, the proportion of R0 resection was even higher; it was achieved in $60 \%$ of patients who were treated with chronomodulated chemotherapy with irinotecan, oxaliplatin, 5- fluorouracil and leucovorin. ${ }^{37}$

In conclusion, the results of our retrospective study showed that the patients with BRAF V600E mutation had worse prognosis than those with wtBRAF, with lower response rates and progressed early during systemic treatment, consequently, with less possibilities to achieve resectability of metastatic disease. The definitive role of the BRAF V600E mutation as a prognostic and predictive factor to response to the anti-EGFR monoclonal antibodies needs to be analyzed in large prospec- tive clinical studies. Different KRAS mutations in codon 12 and 13 and other molecular markers, predictive or prognostic, downstream of EGFR in the RAS/RAF/MAPK pathway, and other effector pathways, are needed to be defined to select the patients, who will benefit from specific systemic therapy in a way of individualized treatment.

\section{References}

1. Cancer in Slovenia 2007. Ljubljana: Institute of Oncology Ljubljana, Epidemiology and Cancer Registry, Cancer Registry of Republic of Slovenia, 2010.

2. Meyerhardt JA, Mayer RJ. Systemic therapy for colorectal cancer. N Eng Med 2005; 352: 476-87.

3. Velenik V, Oblak I, Anderluh F. Quality of life in patients after combined modality treatment of rectal cancer: Report of a prospective phase II study. Radiol Oncol 2008; 42: 207-14.

4. Velenik V. Post-treatment surveillance in colorectal cancer. Radiol Oncol 2010; 44: 135-41.

5. National Comprehensive Cancer Network: NCCN clinical practice guidelines in oncology: colon cancer. V.3.2011 (on line). Avaible: http://www.nccn.org/ profesionals/phisician_gls/PDF/colon.pdf. Accessed April 20, 2011.

6. Advanced colorectal cancer: ESMO clinical practice guidelines for diagnosis, treatment. Ann Oncol 2010; 21: v93-7.

7. Kohne $\mathrm{CH}$. How to integrate molecular targeted agents in the continuum of care. Ann Oncol 2010; 21: vii134-9.

8. Ocvirk J. Advances in the treatment of metastatic colorectal carcinoma. Radiol Oncol 2009; 43: 1-8.

9. Cohen SJ, Cohen RB, Meropol NJ. Targeting signal transducting pathways in colorectal cancer- More than skin deep. J Clin Oncol 2005; 23: 5374-85.

10. Markowitz SD, Bertagnolli MM. Molecular basis of colorectal cancer. N Engl J Med 2009; 361: 2449-60.

11. Artale S, Sartore-Bianchi A, Veronese SM, Gambi V Sarnataro CS, Gambacorta M, et al. Mutations of KRAS and BRAF in primary and matched metastatic sites of colorectal cancer. J Clin Oncol 2008; 26: 4217-9.

12. Etienne- Grimaldi M- C, Formento J- L, Francoual M, François E, Formento $\mathrm{P}$, Renée $\mathrm{N}$, et al. K- Ras mutations and treatment outcome in colorecta cancer patients receiving exclusive fluoropyrimidine therapy. Clin Cancer Res 2008; 14: 4830-5.

13. Ličar A, Cerkovnik P, Ocvirk J, Novaković S. KRAS mutations in Slovene patients with colorectal cancer: frequency, distribution and correlation with the response to treatment. Int J Oncol 2010, 36: 1137-44.

14. De Roock W, Jonker DJ, Di Nicolantonio F, Sartore-Bianchi A, Tu D, Siena $S$, et al. Association of KRAS p.G13D mutation with outcome in patients with outcome with chemotherapy- refractory metastatic colorectal cancer treated with cetuximab. JAMA 2010; 304: 1812-20.

15. Amado RG, Wolf M, Peeters M, Van Cutsem E, Siena S, Freeman DJ, et al. Wild- type KRAS is required for panitumumab efficacy in patients with metastatic colorectal cancer. J Clin Oncol 2008; 26: 1626-34.

16. Roth AD, Tejpar S, Dolorenzi M, Yan P, Fiocca R, Klingbiel D, et al. Prognostic role of KRAS and BRAF in stage II and III resected colon cancer: Results of the translational study on the PETACC-3, EORTC 40993, SAKK 60-00 trial. J Clin Oncol 2010; 28:466-74.

17. Van Cutsem E, Lang I, D'haens G, Moiseyenko V, Zaluski J, Kohne C, et al. The crystal study: Assessment of the predictive value of KRAS status on clinical outcome in patients with $\mathrm{mCRC}$ receiving first-line treatment with cetuximab or cetuximab plus folfiri. [Abstract]. Ann Oncol 2008; 19(Suppl 6): vi17-8.

18. Tol J, Nagtegaal ID, Punt CJA. BRAF mutation in metastatic colorectal cancer N Engl J Med 2009; 361: 98-99. 
19. Ličar A, Cerkovnik P, Novaković S. Distribution of some activating KRAS and BRAF mutations in Slovene patients with colorectal cancer. Med Oncol 2010 Jul 20. [Epub ahead of print]

20. De Roock W, Claes B, Bernasconi D, De Schutter J, Biesmans B, Fountzilas $G$, et al. Effect of KRAS, BRAF, NRAS, and PIK3CA mutations on the efficacy of cetuximab plus chemotherapy in chemotherapy-refractory metastatic colorectal cancer: a retrospective consortium analysis. Lancet Oncol 2010; 11: 753-62.

21. Tejpar S, De Roock W. PIK13CA, BRAF and KRAS mutations and outcome prediction in chemorefractory metastatic colorectal cancer (mCRC) patients treated With EGFR targeting monoclonal antibodies (MoAbs): results of a European Consortium. Eur J Cancer 2009; 7: 322.

22. Lambrechts $D$, De Roock W, Prenen $H$, De Schutter J, Jacobs B, Biesmans $B$, et al. The role of KRAS, BRAF, NRAS and PIK3CA mutations as a marker of resistence of cetuximab in chemorefractory metastatic colorectal cancer. [abstract] J Clin Oncol 2009; 27 (Suppl): 172.

23. Fariña-Sarasqueta A, van Lijnschoten G, Moerland E, Creemers GJ, Lemmens VE, Rutten HJ, et al. The BRAF V600E mutation is an independent prognostic factor for survival in stage II and stage III colon cancer patients. Ann Oncol 2010; 21: 2396-2402.

24. Eisenhauer EA, Therasse P, Bogaerts J, Schwartz LH, Sargent D, Ford R, Dancey J, et al. New response evaluation criteria in solid tumours: Revised RECIST guideline (version 1.1). Eur J Cancer 2009; 45: 228-247.

25. Kohne $\mathrm{CH}$, Rougier $\mathrm{P}$, Stroh $\mathrm{C}$, Schlichting $\mathrm{M}$, Bokemeyer $\mathrm{C}$, Van Cutsem $\mathrm{E}$, et al. Cetuximab with chemotherapy as 1st- line treatment for metastatic colorectal cancer: A meta- analysis of the CRYSTAL and OPUS studies according to KRAS and BRAF mutation status. ASCO GI 2010 [abstract 406]

26. Bookemeyer $\mathrm{C}$, Kohne $\mathrm{CH}$, Rougier $\mathrm{P}$, Stroh C, Schlichting M, Van Cutsem E, et al. Cetuximab with chemotherapy as 1st- line treatment for metastatic colorectal cancer: Analysis of the CRYSTAL and OPUS studies according to KRAS and BRAF mutation status [abstract]. J Clin Oncol 2010; 28 (Suppl): 3506.

27. Tejpar S, Bukemeyer C, Celik I, Schlichting M, Sartorius U, Van Cutsem E, et al. Influence of KRAS G13D mutations on outcome in patients with metastatic colorectal cancer (mCRC) treated with first- line chemotherapy with or without cetuximab. ASCO 2011 [abstract 3511].

28. Loupakis F, Ruzzo A, Cremolini C, Vincenzi B, Salvatore L, Santini D, et al. KRAS codon 61,146 and BRAF mutations predict resistence to cetuximab + irinotecan in KRAS codon 12 and 13 wild-type metastatic colorectal cancer. Br J Cancer 2009; 101: 715-21.

29. Smith G, Bounds R, Wolf H, Steele RJ, Carey FA, Wolf CR, et al. Activating K-ras mutations outwith 'hotspot' codons in sporadic colorectal tumoursimplications for personalised cancer medicine. Br J Cancer 2010; 102: 1-11.

30. Okines A, Del Puerto O, Cunningham D, Chau I, Van Cutsem E, Saltz L, et al. Surgery with curative-intent in patients treated with first-line chemotherapy plus bevacizumab for metastatic colorectal cancer First BEAT and the randomised phase-III NO16966 trial. Br J Cancer 2009; 101: 1033-8.

31. Terrebonne E, Smith D, Becouarn Y, Michel P, Guimbaud M, Fourrier- Reglat $A$, et al. Resection of colorectal cancer (CRC) metastases after bevacizumab (BV) treatment for first-line therapy: results of the ETNA cohort study (abstract 3594). J Clin Oncol 2010; 28: (Suppl.):15s.

32. Ocvirk J, Rebersek M, Boc M. Bevacizumab in first- line therapy of metastatic colorectal cancer: A retrospective comparison of FOLFIRI and XELIRI. Anticancer Res 2011; 31: 1777-82.

33. Wong R, Cunningham D, Barbachano $Y$, et al. A multicentric study of capecitabine, oxaliplatin plus bevacizumab as perioperative treatment of patients with poor- risk colorectal liver- only metastases not selected for upfront resection. Ann Oncol 2011; 22: 2042-8.

34. Van Cutsem E, Kohne CH, Hitre E, Zaluski J, Chang Chien CR, Makhson A, et al. Cetuximab and chemotherapy as initial treatment for metastatic colorectal cancer. N Engl J Med 2009; 360: 1408-17.

35. Bokemeyer C, Bondarenko I, Makhson A, Hartmann JT, Aparicio J, de Braud $F$, et al. Fluorouracil, leucovorin, and oxaliplatin with or without cetuximab in the first- line treatment of metastatic colorectal cancer. J Clin Oncol 2009; 27: $663-71$

36. Folprecht G, Gruenberger T, Bechstein WO, Raab HR, Lordick F, Hartmann $\mathrm{JT}$, et al. Tumour response and secondary resectability of colorectal liver metastases following neoadjuvant chemotherapy with cetuximab: the CELIM randomised phase 2 trial. Lancet Oncol 2010; 1: 38-47.
37. Garufi C, Torsello A, Tumolo S, Ettorre GM, Zeuli M, Campanella C, et al. Cetuximab plus chronomodulated irinotecan, 5- fluorouracil, leucovorin and oxaliplatin as neoadjuvant chemotherapy in colorectal liver metastases: POCHER trial. Br J Cancer 2010; 103: 1-6. 